\title{
Control sets of linear systems on Lie groups
}

\author{
Adriano Da Silva* \\ Instituto de Matemática \\ Universidade Estadual de Campinas \\ Cx. Postal 6065, 13.081-970 Campinas-SP, Brasil \\ Víctor Ayala ${ }^{\dagger}$ \\ Instituto de Alta Investigación \\ Universidad de Tarapacá \\ Casilla 7D, Arica, Chile \\ and \\ Guilherme Zsigmond \\ Universidad Católica del Norte \\ Av. Angamos 0610, Antofagasta, Chile
}

September 5, 2018

\begin{abstract}
Like in the classical linear Euclidean system, we want to characterize for a linear system on a connected Lie group $G$ its control set with nonempty interior $\mathcal{C}$ that contains the identity of $G$. We show that many topological properties of $\mathcal{C}$ are intrinsically connected with the eigenvalues of the derivation $\mathcal{D}$ associated to the drift $\mathcal{X}$. In particular, we prove that if $G$ is a solvable Lie group or if the Lie subgroup $G^{0}=\left\langle\exp \left(\mathfrak{g}^{0}\right)\right\rangle$ is compact, where $\mathfrak{g}^{0}=\bigoplus_{\alpha}\left\{\mathfrak{g}_{\alpha} ; \operatorname{Re}(\alpha)=0\right\}$, then $\mathcal{C}$ is the only control set with nonempty interior of the linear system. Here $\mathfrak{g}_{\alpha}$ stands for the generalized eigenspace associated to the eigenvalue $\alpha$. Furthermore, for nilpotent Lie groups we characterize when the control set $\mathcal{C}$ is bounded. In particular, if $G$ is a nilpotent simply connected Lie group, a linear system on $G$ admits a bounded control set only if $\mathcal{D}$ is hyperbolic.
\end{abstract}

Key words. control sets, linear systems, Lie groups

2010 Mathematics Subject Classification. 16W25; 93B05; 93C05

\footnotetext{
*Supported by Fapesp grant $n^{o}$ 2013/19756-8

${ }^{\dagger}$ Supported by Proyecto Fondecyt $n^{\circ} 1150292$. Conicyt, Chile.
} 


\section{Introduction}

Throughout the paper $G$ stands for a connected Lie group with Lie algebra $\mathfrak{g}$ and dimension $d$. In 2 the authors introduced the class of linear system on $G$, determined by the family of differential equations

$$
\Sigma: \dot{g}(t)=\mathcal{X}(g(t))+\sum_{j=1}^{m} u_{j}(t) X^{j}(g(t)),
$$

here the drift $\mathcal{X}$ with flow $\left(\varphi_{t}\right)_{t \in \mathbb{R}}$ is called a linear vector field which is associated to a $\mathfrak{g}$-derivation $\mathcal{D}$ through the formula

$$
\left(d \varphi_{t}\right)_{e}=\mathrm{e}^{t \mathcal{D}} \quad \text { for all } \quad t \in \mathbb{R} .
$$

The vector fields $X^{j}$ are right invariant and $u \in \mathcal{U} \subset L^{\infty}\left(\mathbb{R}, \Omega \subset \mathbb{R}^{m}\right)$ is the class of admissible controls where $\Omega \subset \mathbb{R}^{m}$ is a compact, convex subset with $0 \in \operatorname{int} \Omega$.

In 9 the author shows that this class of system is also important in applications. Actually, it is shown there that an arbitrary affine control systems on a manifold $M$ which dynamic generate a finite Lie algebra is equivalent to an invariant control systems or a linear control system on a homogeneous space.

On the other hand, it is very well known that the classical linear system on the Euclidean space $\mathbb{R}^{d}$ is one of the most relevant control systems and it can be written as

$$
\dot{x}(t)=A x(t)+\sum_{j=1}^{m} u_{j}(t) b^{j}, \quad b^{j} \in \mathbb{R}^{d} \text { and } u \in \mathcal{U} .
$$

Here $A \in \operatorname{gl}(d, \mathbb{R})$, the Lie algebra of the real matrices of order $d$. Since $\mathbb{R}^{d}$ is commutative, any constant vector $b^{j}$ is an invariant vector field. Just observe that $e^{t A} \in G L^{+}(d, \mathbb{R})=A u t\left(\mathbb{R}^{d}\right)$. This fact gave the first insight in order to generalize the notion of linear systems from $\mathbb{R}^{d}$ to $G$. In fact, the flow $\left(\varphi_{t}\right)_{t \in \mathbb{R}}$ of $\mathcal{X}$ is a 1-parameter group of $G$-automorphisms, and $\left(\frac{d}{d t}\right)_{t=0}\left(\varphi_{t}\right)$ coincides with the derivation $\mathcal{D}$ associated to $\mathcal{X}$, like in the Euclidean case. Of course the $b^{j}$ bi-invariant vector fields on $\mathbb{R}^{d}$ are the columns of the classical cost matrix $B$.

It was shown in [4 that controllability of the linear systems $\Sigma$ on a Lie group is really an exceptional property. In fact, assume that $G$ is nilpotent and the accessibility set $\mathcal{A}$ from the identity element $e$ of $G$ is an open set. It turns out that

$$
\Sigma \text { is controllable on } G \Leftrightarrow \operatorname{Spec}(\mathcal{D}) \cap \mathbb{R}=\{0\} .
$$

Furthermore, recently the authors in [6] proved that $\operatorname{Spec}(\mathcal{D}) \cap \mathbb{R}=\{0\}$ implies controllability for any Lie group with finite semisimple center, that is, any Lie group that admits a maximal semisimple Lie subgroup with finite center.

To understand the controllability behavior of linear systems on Lie groups, we need to approach the problem in a more realistic way. We turn our attention to 
the maximal subsets of $G$ where controllability of the system holds, means, the control sets.

Like in the classical linear system in this paper we characterize the control set with nonemtpy interior of (11) around the identity. As we were expected, many topological properties of $\mathcal{C}$ are intrinsically connected with the eigenvalues of the derivation $\mathcal{D}$ associated to the drift $\mathcal{X}$.

In particular, we prove that if $G$ is a solvable Lie group or if $G^{0}$ is a compact subgroup

$$
\mathcal{C}=\operatorname{cl}(\mathcal{A}) \cap \mathcal{A}^{*}
$$

is the only control set of the linear system $\Sigma$. Here, $\mathcal{A}$ and $\mathcal{A}^{*}$ denote the reachable (controllable) set of the identity element $e$ of $G$ respectively. Furthermore,

$$
\mathcal{C} \text { is bounded } \Rightarrow G^{0}, \operatorname{cl}\left(\mathcal{A}_{G^{-}}\right) \text {and } \operatorname{cl}\left(\mathcal{A}_{G^{+}}^{*}\right) \text { are compact sets, }
$$

where $\mathcal{A}_{G^{-}}\left(\mathcal{A}_{G^{+}}^{*}\right)$ is the intersection of $\mathcal{A}\left(\mathcal{A}^{*}\right)$ with the stable (unstable) manifold of $\varphi$. For the nilpotent case the converse is true. In particular, if $G$ is a nilpotent simply connected Lie group we show that

$\mathcal{C}$ is bounded $\Leftrightarrow \operatorname{cl}\left(\mathcal{A}_{G^{-}}\right)$and $\operatorname{cl}\left(\mathcal{A}_{G^{+}}^{*}\right)$ are compacts and $\mathcal{D}$ is hyperbolic.

The paper is structured as follows: Section 2 introduces the notion of affine control systems and its control sets on an arbitrary differentiable manifold. We state here basic properties of control sets with nonempty interior. Furthermore, we define linear vector field and linear systems. Associated with any $\mathfrak{g}$-derivation there are several Lie algebras and their corresponding Lie groups, connected with the reachable and controllable sets of the system. We take care of this decomposition here. In Section 3 we analyze the control sets of the linear system (11). By a general result from [3, it follows that around the identity of $G$ there exists one of these possible sets. Then we focus our attention on its properties. In particular, we establish necessary and sufficient conditions to decide whenever this set is invariant. On the other hand, we analyze under which circumstances the control set is the whole $G$ and when it is bounded. At the end of Section 3 we prove our main result. We show that the control set around the identity is the only maximal set of approximate controllability for Lie groups where we can decompose $G$ as the product of the subgroups associated with the real parts of the eigenvalues of $\mathcal{D}$.

\section{Preliminaries}

In this Section we state the definitions and main results concerning to affine control system, control sets, linear vector field the associated subalgebras and the corresponding subgroups. For more on the subjects the reader could consult [1], [2], 3], 4], 7], [8] and [9]. 


\subsection{Affine control systems and its control sets}

Let $M$ be a $d$ dimensional smooth manifold. By an affine control system in $M$ we understand the family of ordinary differential equations

$$
\dot{x}(t)=f^{0}(x(t))+\sum_{j=1}^{m} u_{j}(t) f^{j}(x(t)), \quad u=\left(u_{1}, \ldots, u_{m}\right) \in \mathcal{U}
$$

where $f^{j} \in X^{\infty}(M), j=0,1, \ldots, m$ are arbitrary $\mathcal{C}^{\infty}$ vector fields on $M$.

As usual, the family $\mathcal{U} \subset L^{\infty}\left(\mathbb{R}, \Omega \subset \mathbb{R}^{m}\right)$ is the class of restricted admissible control functions where $\Omega \subset \mathbb{R}^{m}$ with $0 \in \operatorname{int} \Omega$, is a compact and convex set called the control range of the system.

Since our work focus on Lie groups we assume that the vector fields $f^{j}, j=$ $0,1, \ldots, m$ are analytical. Therefore, for each control function $u \in \mathcal{U}$ and each initial value $x \in M$ there exists an unique solution $\phi(t, x, u)$ defined on an open interval containing $t=0$, satisfying $\phi(0, x, u)=x$. Note that in general $\phi(t, x, u)$ is only a solution in the sense of Carathéodory, i.e., a locally absolutely continuous curve satisfying the corresponding differential equation almost everywhere. Without lost of generality we assume that any solution can be extended to the whole real line. Hence, we obtain a mapping

$$
\phi: \mathbb{R} \times M \times \mathcal{U} \rightarrow M, \quad(t, x, u) \mapsto \phi(t, x, u),
$$

satisfying the cocycle property

$$
\phi(t+s, x, u)=\phi\left(t, \phi(s, x, u), \Theta_{s} u\right)
$$

for all $t, s \in \mathbb{R}, x \in M, u \in \mathcal{U}$. Here for any $t \in \mathbb{R}$ the map $\Theta_{t}$ is the shift flow on $\mathcal{U}$ defined by

$$
\left(\Theta_{t} u\right)(s):=u(t+s) .
$$

Instead of $\phi(t, x, u)$ we usually write $\phi_{t, u}(x)$. Note that smoothness of the vector fields $f^{0}, f^{1}, \ldots, f^{m}$ implies the smoothness of $\phi_{t, u}$. Moreover, it follows directly from the cocycle property that

i) the inverse of the diffeomorphism $\phi_{t, u}$ exists and it is given by $\phi_{-t, \Theta_{t} u}$

ii) the fact that for any $t>0, \phi_{t, u}(g)$ depends just on $\left.u\right|_{[0, t]}$ implies that

$$
\phi_{t, u_{1}}\left(\phi_{s, u_{2}}(g)\right)=\phi_{t+s, u}(g)
$$

where $u \in \mathcal{U}$ is defined through concatenation by

$$
u(\tau)=\left\{\begin{array}{c}
u_{1}(\tau) \text { for } \tau \in[0, s] \\
u_{2}(\tau-s) \text { for } \tau \in[s, t+s] .
\end{array}\right.
$$


For any $x \in M$ and $\tau>0$ the sets

$$
\begin{aligned}
& \mathcal{A}_{\leq \tau}(x):=\left\{y \in M: \exists u \in \mathcal{U}, t \in[0, \tau] \text { with } y=\phi_{t, u}(x)\right\} \\
& \mathcal{A}_{\tau}(x):=\left\{y \in M: \exists u \in \mathcal{U}, \quad y=\phi_{\tau, u}(x)\right\} \\
& \mathcal{A}(x):=\bigcup_{\tau>0} \mathcal{A}_{\leq \tau}(x),
\end{aligned}
$$

are the set of reachable point from $x$ up to time $\tau$, the set of reachable points from $x$ at time $\tau$ and the reachable set of $x$, respectively. In the same way, for any $\tau>0$ the sets

$$
\begin{aligned}
& \mathcal{A}_{\leq \tau}^{*}(x):=\left\{y \in M: \exists u \in \mathcal{U}, t \in[0, \tau] ; \quad \phi_{t, u}(y)=x\right\} \\
& \mathcal{A}_{\tau}^{*}(x):=\left\{y \in M: \exists u \in \mathcal{U}, \quad y=\phi_{\tau, u}(x)\right\} \\
& \mathcal{A}^{*}(x):=\bigcup_{\tau>0} \mathcal{A}_{\leq \tau}^{*}(x),
\end{aligned}
$$

are called the set of controllable points to $x$ within time $\tau$, the set of controllable points to $x$ in time $\tau$ and the controllable set of $x$, respectively.

2.1 Definition: We say that the system (2)

$i)$ is locally accessible at $x$ if for all $\tau>0$ the sets $\mathcal{A}_{\leq \tau}(x)$ and $\mathcal{A}_{\leq \tau}^{*}(x)$ have nonempty interior

ii) is locally accessible if it is locally accessible at every $x \in M$

iii) satisfy the Lie algebra rank condition (LARC) if $\mathcal{L}(x)=T_{x} M$ for any $x \in M$, where

$\mathcal{L}$ is the smallest $\mathfrak{g}$-subalgebra containing any $f^{j}, j=0,1, \ldots, m$.

Moreover, the system is locally accessible at $x \in M$ if it satisfies the Lie algebra rank condition at the point $x$.

On the other hand, a more convenient approach to understand the controllability behavior of a linear system comes from the notion of control set.

2.2 Definition: $A$ nonempty set $\mathcal{C} \subset M$ is called a control set of the affine system (2) if it is

i) controlled invariant, that is, for every $x \in M$ there exists $u \in \mathcal{U}$ such that $\phi(\mathbb{R}, x, u) \subset \mathcal{C}$

ii) approximate controllable, that is, $\mathcal{C} \subset \operatorname{cl}(\mathcal{A}(x))$ for every $x \in \mathcal{C}$

iii) is maximal with properties $(i)$ and $(i i)$ 
By Proposition 3.2.4 of [3] , a set $\mathcal{C}$ that is maximal with respect to the property (ii) above and satisfies int $\mathcal{C} \neq \emptyset$ is a control set.

A control set $\mathcal{C}$ is said to be invariant in positive time if $\phi_{t, u}(\mathcal{C}) \subset \mathcal{C}$ for any $t>0$ and $u \in \mathcal{U}$. In the same way, we say that $\mathcal{C}$ is invariant in negative time if $\phi_{-t, u}(\mathcal{C}) \subset \mathcal{C}$ for any $t>0$ and $u \in \mathcal{U}$.

The next proposition summarizes the main properties of control sets. Its proof can be mainly found in [3] Theorem 3.1.5.

2.3 Proposition: Assume that the system (2) is locally accessible and let $\mathcal{C}$ be a control set with nonempty interior. It holds

1. $\mathcal{C}$ is connected and $\operatorname{cl}(\operatorname{int} \mathcal{C})=\operatorname{cl}(\mathcal{C})$

2. $\operatorname{int} \mathcal{C} \subset \mathcal{A}(x)$ for any $x \in \mathcal{C}$. For any $y \in \operatorname{int} \mathcal{C}$

$$
\mathcal{C}=\operatorname{cl}(\mathcal{A}(y)) \cap \mathcal{A}^{*}(y) .
$$

In particular the system is controllable on int $\mathcal{C}$.

3. Assume that $\phi_{t, u}(x)$ is a periodic trajectory, that is, $\phi_{t+s, u}(x)=\phi_{t, u}(x)$ for some $s>0$ and all $t \in \mathbb{R}$. Therefore, if $x \in \operatorname{int} \mathcal{C}$ then $\phi_{t, u}(x) \in \operatorname{int} \mathcal{C}$ for all $t \in \mathbb{R}$.

4. $\mathcal{C}$ is closed $\Leftrightarrow \mathcal{C}$ is invariant in positive time $\Leftrightarrow \mathcal{C}=\operatorname{cl}(\mathcal{A}(g))$ for any $g \in \mathcal{C}$;

5. $\mathcal{C}$ is open $\Leftrightarrow \mathcal{C}$ is invariant in negative time $\Leftrightarrow \mathcal{C}=\mathcal{A}^{*}(g)$ for any $g \in \mathcal{C}$;

\subsection{Linear vector fields and decompositions}

Let $G$ be a connected Lie group with Lie algebra $\mathfrak{g}$.

2.4 Definition: $A$ vector field $\mathcal{X}$ on $G$ is said to be linear if its flows $\left(\varphi_{t}\right)_{t \in \mathbb{R}}$ is a 1-parameter group of $G$-automorphisms.

Certainly, the vector field $\mathcal{X}$ is complete. Furthermore, one can associate to $\mathcal{X}$ a derivation $\mathcal{D}$ of $\mathfrak{g}$ defined by

$$
\mathcal{D} Y=-[\mathcal{X}, Y](e), \text { for all } Y \in \mathfrak{g} .
$$

The relation between $\varphi_{t}$ and $\mathcal{D}$ is given by the formula

$$
\left(d \varphi_{t}\right)_{e}=\mathrm{e}^{t \mathcal{D}} \quad \text { for all } t \in \mathbb{R}
$$

which implies that

$$
\varphi_{t}(\exp Y)=\exp \left(\mathrm{e}^{t \mathcal{D}} Y\right), \text { for all } t \in \mathbb{R}, Y \in \mathfrak{g} .
$$


On the other hand, if the group is simply connected any derivation $\mathcal{D}$ has an associated linear vector field $\mathcal{X}=\mathcal{X}^{D}$ through the same formula above. For connected Lie groups the same is true when $\mathcal{D} \in \mathfrak{a} \mathfrak{u t}(G)$ the Lie algebra of $\operatorname{Aut}(G)$ the Lie group of $G$-automorphism (see [2]).

Next we explicitly some decomposition of $\mathfrak{g}$ and $G$ induced by the derivation $\mathcal{D}$. Let us consider the generalized eigenspaces of $\mathcal{D}$ given by

$$
\mathfrak{g}_{\alpha}=\left\{X \in \mathfrak{g}:(\mathcal{D}-\alpha)^{n} X=0 \text { for some } n \geq 1\right\}
$$

where $\alpha$ is an eigenvalue of $\mathcal{D}$.

It turns out that $\left[\mathfrak{g}_{\alpha}, \mathfrak{g}_{\beta}\right] \subset \mathfrak{g}_{\alpha+\beta}$ when $\alpha+\beta$ is an eigenvalue of $\mathcal{D}$ and zero otherwise. This fact allow to us to decompose $\mathfrak{g}$ as

$$
\mathfrak{g}=\mathfrak{g}^{+} \oplus \mathfrak{g}^{0} \oplus \mathfrak{g}^{-}
$$

where

$\mathfrak{g}^{+}=\bigoplus_{\alpha: \operatorname{Re}(\alpha)>0} \mathfrak{g}_{\alpha}, \quad \mathfrak{g}^{0}=\bigoplus_{\alpha: \operatorname{Re}(\alpha)=0} \mathfrak{g}_{\alpha} \quad$ and $\quad \mathfrak{g}^{-}=\bigoplus_{\alpha: \operatorname{Re}(\alpha)<0} \mathfrak{g}_{\alpha}$.

It is easy to see that $\mathfrak{g}^{+}, \mathfrak{g}^{0}, \mathfrak{g}^{-}$are Lie algebras and $\mathfrak{g}^{+}, \mathfrak{g}^{-}$are nilpotent (see Proposition 3.1 of [11]).

We denote by $G^{+}, G^{-}, G^{0}, G^{+, 0}$, and $G^{-, 0}$ the connected Lie subgroups of $G$ with Lie algebras $\mathfrak{g}^{+}, \mathfrak{g}^{-}, \mathfrak{g}^{0}, \mathfrak{g}^{+, 0}:=\mathfrak{g}^{+} \oplus \mathfrak{g}^{0}$ and $\mathfrak{g}^{-, 0}:=\mathfrak{g}^{-} \oplus \mathfrak{g}^{0}$ respectively.

\subsection{Linear systems on Lie groups}

A linear system on a Lie group $G$ is a control-affine system

$$
\dot{g}(t)=\mathcal{X}(g(t))+\sum_{j=1}^{m} u_{j}(t) X^{j}(g(t)),
$$

where the drift vector field $\mathcal{X}$ is a linear vector field, $X^{j}$ are right invariant vector fields and $u=\left(u_{1}, \cdots, u_{m}\right) \in \mathcal{U}$ as before.

For a given $g \in G, u \in \mathcal{U}$ and $t \in \mathbb{R}$ the solution of the linear system (7) starting at $g$ reads as

$$
\phi_{t, u}(g)=L_{\phi_{t, u}}\left(\varphi_{t}(g)\right)=\phi_{t, u} \varphi_{t}(g),
$$

where $\phi_{t, u}=\phi_{t, u}(e)$ is the solution of (77) starting at the identity element $e \in G$ (see for instance [5]).

Let us denote by $\mathcal{A}_{\leq \tau}, \mathcal{A}_{\tau}$ and $\mathcal{A}$ the sets $\mathcal{A}_{\leq \tau}(e), \mathcal{A}_{\tau}(e)$ and $\mathcal{A}(e)$, respectively. For any $u \in \mathcal{U}$ it follows from equation (8) that the solutions of the linear system (77) satisfy $\phi_{t, u}^{-1}=\phi_{-t, \Theta_{t} u}$. Therefore,

$$
\mathcal{A}_{\tau}^{*}=\varphi_{-\tau}\left(\mathcal{A}_{\tau}^{-1}\right) .
$$

The next proposition states the main properties of the reachable sets of linear systems, its proof can be found in [7, Proposition 2. 
2.5 Proposition: With the previous notations it holds:

1. $\tau>0 \Rightarrow \mathcal{A}_{\tau}=\mathcal{A}_{\leq \tau}$

2. $0 \leq \tau_{1} \leq \tau_{2} \Rightarrow \mathcal{A}_{\tau_{1}} \leq \mathcal{A}_{\tau_{2}}$

3. $g \in G \Rightarrow \mathcal{A}_{\tau}(g)=\mathcal{A}_{\tau} \varphi_{\tau}(g)$

4. $\tau_{1}, \tau_{2} \geq 0 \Rightarrow \mathcal{A}_{\tau_{1}+\tau_{2}}=\mathcal{A}_{\tau_{1}} \varphi_{\tau_{1}}\left(\mathcal{A}_{\tau_{2}}\right)=\mathcal{A}_{\tau_{2}} \varphi_{\tau_{2}}\left(\mathcal{A}_{\tau_{1}}\right)$

The next result (Lemma 3.1 of [4) shows that the accessible set $\mathcal{A}$ is invariant by right translations of elements whose $\mathcal{X}$-orbits are contained in $\mathcal{A}$.

2.6 Lemma: Let $g \in \mathcal{A}$ and assume that $\varphi_{t}(g) \in \mathcal{A}$ for any $t \in \mathbb{R}$. Then $\mathcal{A} \cdot g \subset \mathcal{A}$.

2.7 Remark: Using the above lemma it follows that

$$
\text { if } \varphi_{t}(g) \in \operatorname{cl}(\mathcal{A}) \text { for any } t \in \mathbb{R} \Rightarrow \operatorname{cl}(\mathcal{A}) \cdot g \subset \operatorname{cl}(\mathcal{A}) \text {. }
$$

In order to extend the controllability results in [4 from solvable groups to more general Lie groups, the authors in [6] introduced the following notion

2.8 Definition: Let $G$ be a connected Lie group. We say that the Lie group $G$ has finite semisimple center if all semisimple Lie subgroups of $G$ have finite center.

For such class of Lie groups we have the following result (Theorem 3.9 of []).

2.9 Proposition: Let $G$ be a connected Lie group with finite semisimple center. If $\mathcal{A}$ is open, then $G^{+, 0} \subset \mathcal{A}$ and $G^{-, 0} \subset \mathcal{A}^{*}$.

2.10 Remark: We should notice that a sufficient condition to get $G^{+, 0} \subset \mathcal{A}$ in Theorem 3.9 of $[6$ is the fact that

$$
e \in \operatorname{int} \mathcal{A}_{\tau_{0}}, \text { for some } \tau_{0}>0 .
$$

However, by Lemma 4.5.2 of 3 we know that this condition is equivalent to $\mathcal{A}$ being open.

2.11 Remark: We should also notice that the condition on the openness of $\mathcal{A}$ implies, in particular, that the system satisfies the Lie algebra rank condition (see Theorem 3.3 of [2]). 


\section{Control sets of a linear system}

In this section we prove our main result. We start with a proposition which states properties of the subgroups obtained from the $\mathcal{D}$-decomposition.

3.1 Proposition: It holds :

1. $G^{+, 0}=G^{+} G^{0}=G^{0} G^{+}$and $G^{-, 0}=G^{-} G^{0}=G^{0} G^{-}$

2. $G^{+} \cap G^{-}=G^{+, 0} \cap G^{-}=G^{-, 0} \cap G^{+}=\{e\}$

3. $G^{+, 0} \cap G^{-, 0}=G^{0}$

4. All the above subgroups are closed in $G$

5. If $G$ is solvable then

$$
G=G^{+, 0} G^{-}=G^{-, 0} G^{+}
$$

Moreover, the fixed points of $\mathcal{X}$ are in $G^{0}$;

Proof: The proof of items 1. to 5. can be found in [4] Proposition 2.9.

Next we prove the following

3.2 Proposition: If $\mathcal{D}$ is inner and $G^{0}$ is compact, then $G=G^{0}$. Furthermore, the decomposition (10) also holds when we just assume that $G^{0}$ is a compact subgroup.

Proof: Let us assume $\mathcal{D}=\operatorname{ad}(X)$ for some $X \in \mathfrak{g}$. Certainly $X \in \mathfrak{g}^{0}$ and it holds that

$$
\varphi_{t}(g)=\mathrm{e}^{-t X} g \mathrm{e}^{t X}, \quad \text { for any } t \in \mathbb{R} \text { and } g \in G \text {. }
$$

Consequently, if $G^{0}$ is compact, the $\mathcal{X}$-orbit

$$
\mathcal{O}(g)=\left\{\varphi_{t}(g), t \in \mathbb{R}\right\}
$$

is bounded for any $g \in G$, since it is contained in the compact set $K_{g}=G^{0} g G^{0}$. In particular, if $g \in G^{-}$, by the $\mathcal{D}$-invariance of $\mathfrak{g}^{-}$we obtain that $\mathcal{O}(g) \subset$ $G^{-} \cap K_{g}$ is bounded in $G^{-}$.

Considering that $\left.\varphi_{t}\right|_{G^{-}}$is an automorphism of $G^{-}$if follows that

$$
\varrho\left(\varphi_{t}(g), \varphi_{t}(h)\right) \leq\left\|\left(\left.d \varphi_{t}\right|_{G^{-}}\right)_{e}\right\| \varrho(g, h), \quad g, h \in G^{-} \text {and } t \geq 0,
$$

for any left invariant Riemannian metric on $G^{-}$.

On the other hand, since $\left(d \varphi_{t}\right)_{e}=\mathrm{e}^{t \mathcal{D}}$ and $\left.\mathcal{D}\right|_{\mathfrak{g}^{-}}$has only eigenvalues with negative real part there are $c, \mu>0$ such that

$$
\left\|\left(\left.d \varphi_{t}\right|_{G^{-}}\right)_{e}\right\|=\left\|\mathrm{e}^{\left.t \mathcal{D}\right|_{\mathfrak{g}^{-}}}\right\| \leq c^{-1} \mathrm{e}^{-\mu t} \text { for any } t \geq 0
$$


implying that

$$
\varrho\left(\varphi_{t}(g), \varphi_{t}(h)\right) \leq c^{-1} \mathrm{e}^{-\mu t} \varrho(g, h), \quad g, h \in G^{-} \text {and } t \geq 0 .
$$

Consequently

$$
\varrho\left(\varphi_{-t}(g), \varphi_{-t}(h)\right) \geq c \mathrm{e}^{\mu t} \varrho(g, h), \quad g, h \in G^{-}, t \geq 0
$$

which shows that $\mathcal{O}(g)$ is bounded in $G^{-}$if and only, if $g=e$. Therefore, if $G^{0}$ is compact we must have $G^{-}=\{e\}$. Analogously $G^{+}=\{e\}$ and $G=G^{0}$ as stated.

Assume now that $G^{0}$ is a compact subgroup of $G$ and $\mathcal{D}$ is an arbitrary derivation. Let $\mathfrak{r}=\mathfrak{r}(\mathfrak{g})$ stands for the solvable radical of $\mathfrak{g}$ and $R \subset G$ its associated connected solvable Lie subgroup. Since $\mathfrak{r}$ is a $\mathcal{D}$-invariant ideal of $\mathfrak{g}$, we obtain a well induced linear vector field on the semisimple Lie group $G / R$.

Now, any derivation on a semisimple Lie algebra is inner. On the other hand, Lemma 2.3 of 4 give us that

$$
(G / R)^{0}=\pi\left(G^{0}\right) \text { where } \pi: G \rightarrow G / R
$$

is the canonical projection. Therefore, from the compacity of $G^{0}$ that we are assuming we get

$$
G / R=(G / R)^{0}=\pi\left(G^{0}\right) .
$$

Consequently, $G=G^{0} R$. But $R$ is $\varphi$-invariant, then item 5 of the previous proposition shows that

$$
R=R^{+, 0} R^{-}=R^{-, 0} R^{+},
$$

where $R^{+}, R^{-}, R^{0}$ are the connected Lie subgroup of $R$ with Lie algebras

$$
\mathfrak{r} \cap \mathfrak{g}^{+}, \mathfrak{r} \cap \mathfrak{g}^{-}, \mathfrak{r} \cap \mathfrak{g}^{0}
$$

respectively.

Now, by using item 1 of the same proposition we conclude that

$$
G=G^{+, 0} G^{-}=G^{-, 0} G^{+}
$$

as stated.

3.3 Remark: The condition on the compacity of $G^{0}$ can be weakened. In fact, it is enough to ask that the connected components of the Lie subgroup of $G / R$ given by the singularities of the induced linear vector field on $G / R$ are compacts.

Next we show that if $G$ has the decomposition (10), the reachable and controllable sets have also such a decompositions. 
3.4 Lemma: Let us assume that $G$ has finite semisimple center and $\mathcal{A}$ is open. If $G$ has the decomposition (10) then

$$
\mathcal{A}=\mathcal{A}_{G^{-}} G^{+, 0} \quad \text { and } \quad \mathcal{A}^{*}=\mathcal{A}_{G^{+}}^{*} G^{-, 0},
$$

where

$$
\mathcal{A}_{G^{-}}=\mathcal{A} \cap G^{-} \text {and } \mathcal{A}_{G^{+}}^{*}=\mathcal{A}^{*} \cap G^{+} .
$$

Proof: We only show the decomposition for $\mathcal{A}$ since the other case is analogous. By hypothesis, $G^{+, 0} \subset \mathcal{A}$. The $\varphi$-invariance of $G^{+, 0}$ implies by Lemma 2.6 that

$$
\mathcal{A}_{G^{-}} G^{+, 0} \subset \mathcal{A} G^{+, 0} \subset \mathcal{A} .
$$

Reciprocally, let $x \in \mathcal{A}$. By decomposition (10) there are $a \in G^{-}$and $b \in G^{+, 0}$ such that $x=a b$. Moreover,

$$
a=x b^{-1} \in \mathcal{A} b^{-1} \subset \mathcal{A} \Rightarrow a \in \mathcal{A}_{G^{-}} .
$$

Therefore, $\mathcal{A}=\mathcal{A}_{G^{-}} G^{+, 0}$ as desired.

From now we assume that $G$ has finite semisimple center. We notice that there are many Lie groups satisfying this property. Of course, any solvable group and any semisimple Lie group with finite center, like $\mathfrak{s l}(n, \mathbb{R})$, has the finite semisimple center property. But also, the direct or semidirect product between groups with finite semisimple center have the same property. Furthermore, according to the Levi Decomposition Theorem given a Lie algebra $\mathfrak{g}$ there exists a semisimple subalgebra $\mathfrak{s}$ of $\mathfrak{g}$ such that

$$
\mathfrak{g}=\mathfrak{r}(\mathfrak{g}) \oplus \mathfrak{s}
$$

where $\mathfrak{r}(\mathfrak{g})$ is the solvable radical, i.,e., the largest solvable subgroup of $G$.

In particular, if one Levi subgroup of $G$ has finite center, then $G$ has finite semisimple center (see Theorem 4.3 of [10] and corollaries therein).

Let us also assume from now that the reachable set $\mathcal{A}$ is open. Since $0 \in \operatorname{int} \Omega$, Corollary 4.5.11 of [3] assures the existence of a control set $\mathcal{C}$ of the linear system (17) that contains the identity element $e \in G$ in its interior. Our aim here is to analyze the topological properties of this control set and to understand in which cases it is in fact the only control set of (7) with nonempty interior. A first result in this direction is the following

3.5 Theorem: Assume that $G$ has finite semisimple center and the reachable set $\mathcal{A}$ of the linear system $\Sigma$ is open. For the existent control set $\mathcal{C}$ it holds that

1. $\mathcal{C}$ is closed if and only if $\mathcal{A}^{*}=G$

2. $\mathcal{C}$ is open if and only if $\mathcal{A}=G$

3. Furthermore, if $G$ is nilpotent we have 
i) $\mathcal{C}$ is closed if and only if $\mathcal{D}$ has only eigenvalues with nonpositive real part

ii) $\mathcal{C}$ is open if and only if $\mathcal{D}$ has only eigenvalues with nonnegative real part

iii) $\mathcal{C}=G$ if and only if $\mathcal{D}$ has only eigenvalues with zero real part

Proof: 1 . If $\mathcal{A}^{*}=G$ we get

$$
\mathcal{C}=\operatorname{cl}(\mathcal{A}) \cap \mathcal{A}^{*}=\operatorname{cl}(\mathcal{A})
$$

showing that $\mathcal{C}$ is closed. Reciprocally, if $\mathcal{C}$ is closed, by item 4. of Proposition 2.3 it follows that $\mathcal{C}=\operatorname{cl}(\mathcal{A}(g))$ for any $g \in G$ and in particular $\mathcal{C}=\operatorname{cl}(\mathcal{A})$.

By item 2. of the same Proposition we must have $\mathcal{A} \subset \mathcal{A}^{*}$. Since $G^{+} \subset \mathcal{A}$ Lemma 2.6 shows that

$$
B=G^{+} G^{-, 0} \subset \mathcal{A}^{*} G^{-, 0} \subset \mathcal{A}^{*} .
$$

Since $B$ is $\varphi$-invariant, Lemma 2.6 implies also that $B^{n} \subset \mathcal{A}^{*}$. Moreover, $B$ is a neighborhood of $e \in G$ and since $G$ is connected we get

$$
G=\bigcup_{n \in \mathbb{N}} B^{n} \subset \mathcal{A}^{*} \Rightarrow \mathcal{A}^{*}=G .
$$

2. If $\mathcal{A}=G$ we obtain

$$
\mathcal{C}=\operatorname{cl}(\mathcal{A}) \cap \mathcal{A}^{*}=\mathcal{A}^{*}
$$

showing in particular that $\mathcal{C}$ is open. Conversely, let us assume that $\mathcal{C}=\mathcal{A}^{*}(g)$ for any $g \in \mathcal{C}$. By item 2. of Proposition 2.3 we have that int $\mathcal{C} \subset \mathcal{A}(g)$ for any $g \in \mathcal{C}$. In particular, since $\mathcal{C}=\mathcal{A}^{*}$ and $\mathcal{A}^{*}$ is open we get $\mathcal{A}^{*} \subset \mathcal{A}$. Moreover, since $G^{-} \subset \mathcal{A}^{*}, G^{+, 0} \subset \mathcal{A}$ and both are $\varphi$-invariant, we obtain

$$
B=G^{+} G^{-, 0}=G^{+, 0} G^{-} \subset \mathcal{A} G^{-} \subset \mathcal{A} .
$$

As before, this fact allows to us to conclude that $\mathcal{A}=G$ as desired.

3. If $G$ is nilpotent, Proposition 4.4 of [4] assures that $\mathcal{A}^{*}=G$ if and only if $G=G^{-, 0}$ and $\mathcal{A}=G$ if and only if $G=G^{+, 0}$ which proves item i) and ii). Item iii) follows from Theorem 4.5 of [4].

The above theorem implies in particular that if $\mathcal{C}$ is an invariant control set, it is the only invariant one. In fact,

3.6 Corollary: Assume that $G$ has finite semisimple center and the reachable set $\mathcal{A}$ of the linear system $\Sigma$ is open. If the existent control set $\mathcal{C}$ is invariant, then it is the only invariant one.

Proof: Let us prove the case where $\mathcal{C}$ is invariant in positive time. By item 4 . of Proposition 2.3 that situation happens if and only if $\mathcal{C}$ is closed and by the theorem above, if and only if $\mathcal{A}^{*}=G$. 
If $\widetilde{\mathcal{C}}$ is another closed control set, for any $g \in \widetilde{\mathcal{C}} \subset \mathcal{A}^{*}$ there are $\tau>0, u \in \mathcal{U}$ such that $\phi_{\tau, u}(g)=e$. Moreover, by the invariance of $\widetilde{\mathcal{C}}$ we must have

$$
e=\phi_{\tau, u}(g) \in \phi_{\tau, u}(\widetilde{\mathcal{C}}) \subset \widetilde{\mathcal{C}} .
$$

Showing that $e \in \mathcal{C} \cap \widetilde{\mathcal{C}}$.

Since two control sets with nonempty intersection must coincide we must have $\mathcal{C}=\widetilde{\mathcal{C}}$. Then, $\mathcal{C}$ is the only invariant one.

3.7 Remark: We should notice that the above results show us how restrictive is the condition for a linear system to have an invariant control set. A linear system admits a positive/negative invariant control set if and only if the whole group $G$ is controllable to/reachable from the identity. Furthermore, in [7] the author shows that under the LARC condition, the positive orbit $\mathcal{A}$ of an unrestricted linear control systems is a semigroup if and only if $\mathcal{A}=G$. Here, unrestricted means (7) with $\Omega=\mathbb{R}^{m}$.

\subsection{Bounded control sets}

In this section our interest is to search for conditions when our control set $\mathcal{C}$ is bounded. As before we are assuming that $G$ is a connected Lie group with finite semisimple center and that the linear system on $G$ is such that the reachable set $\mathcal{A}$ is open.

Let us consider as before the sets

$$
\mathcal{A}_{G^{-}}=\mathcal{A} \cap G^{-} \text {and } \mathcal{A}_{G^{+}}^{*}=\mathcal{A}^{*} \cap G^{+}
$$

Since $\mathcal{A}_{G^{-}}, \mathcal{A}_{G^{+}}^{*}$ and $G^{0}$ are contained in $\mathcal{A} \cap \mathcal{A}^{*}$, if the control set $\mathcal{C}$ is bounded we get

$$
\operatorname{cl}\left(\mathcal{A}_{G^{-}}\right), \operatorname{cl}\left(\mathcal{A}_{G^{+}}^{*}\right) \quad \text { and } \quad G^{0} \text { are compact sets. }
$$

Next we show that in some cases the compacity of such sets imply that $\mathcal{C}$ is bounded.

3.8 Theorem: Let us assume that $G$ is semisimple or nilpotent. If $\operatorname{cl}\left(\mathcal{A}_{G^{-}}\right)$, $\operatorname{cl}\left(\mathcal{A}_{G^{+}}^{*}\right)$ and $G^{0}$ are compact subsets of $G$ then $\mathcal{C}$ is bounded.

Proof: If $G$ is semisimple, the result follows direct from Proposition 3.2 since in this case $G=G^{0}$. For the nilpotent case we prove by induction on the dimension of $G$.

If $\operatorname{dim} G=1$ then $G$ is Abelian and by Lemma 3.4 we have that

$$
\mathcal{A} \cap \mathcal{A}^{*}=\mathcal{A}_{G^{-}} G^{-, 0} \cap \mathcal{A}_{G^{+}}^{*} G^{+, 0}=\mathcal{A}_{G^{-}} G^{0} \mathcal{A}_{G^{+}}^{*} .
$$

Considering that $\mathcal{A} \cap \mathcal{A}^{*}$ is dense in $\mathcal{C}$ we get

$$
\mathcal{C} \subset \operatorname{cl}\left(\mathcal{A}_{G^{-}}\right) G^{0} \operatorname{cl}\left(\mathcal{A}_{G^{+}}^{*}\right)
$$


which by our assumptions is a compact set, implying that $\mathcal{C}$ is a bounded control set.

Let us assume that $G$ is a nilpotent Lie group with dimension $n$.

Let $Z_{G}$ be the center of $G$ and $\left(Z_{G}\right)_{0}$ its connected component of the identity. Since $\left(Z_{G}\right)_{0}$ is $\varphi$-invariant and Abelian, we have by item 5 . of Proposition 3.1 that $\left(Z_{G}\right)_{0}=Z^{+} Z^{0} Z^{-}$. Moreover $Z^{+}, Z^{0}$ and $Z^{-}$are $\varphi$-invariant normal subgroups of $G$. Since $G$ is nilpotent, $\left(Z_{G}\right)_{0}$ is nontrivial and consequently at least one of the subgroups $Z^{+}, Z^{0}$ or $Z^{-}$is nontrivial.

Let us analyze the case when $\{e\} \varsubsetneqq Z^{+}$, the other cases are analogous. Consider the connected nilpotent Lie group $H=G / Z^{+}$. By the $\varphi$-invariance of $Z^{+}$we get an induced linear system on $H$ (see Proposition 4 of [9]) that satisfy

$$
\pi\left(\phi_{t, u}^{G}(g)\right)=\phi_{t, u}^{H}(\pi(g))
$$

where $\pi: G \rightarrow H$ is the canonical projection.

The above equation gives us that $\pi(\mathcal{A})$ and $\pi\left(\mathcal{A}^{*}\right)$ are the reachable and controllable sets of the identity in $H$ respectively. Considering that

$$
\operatorname{dim} H=\operatorname{dim} G-\operatorname{dim} Z^{+}<n
$$

by hypothesis it follows that

$$
\mathcal{C}^{H}=\operatorname{cl}(\pi(\mathcal{A})) \cap \pi\left(\mathcal{A}^{*}\right) \text { is a bounded control set. }
$$

Since $\pi$ is an open map, there exists a compact set $K \subset G$ such that

$$
\pi(\mathcal{C}) \subset \mathcal{C}^{H} \subset \pi(K) \text { implying that } \mathcal{C} \subset K Z^{+} .
$$

Since $G^{0}$ is compact, $G$ has the decomposition (10) and we can assume without lost of generality that

$$
K=K^{+} K^{-, 0}, K^{+} \text {and } K^{-, 0} \text { are compact subsets of } G^{+} \text {and } G^{-, 0}
$$

respectively.

Take $x \in \mathcal{A} \cap \mathcal{A}^{*}$. There are

$$
k_{1} \in K^{+}, k_{2} \in K^{-, 0} \text { and } z \in Z^{+} ; x=k_{1} k_{2} z .
$$

But $Z^{+} \subset\left(Z_{G}\right)_{0}$ so we obtain $x=\left(z k_{1}\right) k_{2}$. Since by Lemma 3.4 we have $\mathcal{A}^{*}=\mathcal{A}_{G^{+}}^{*} G^{-, 0}$ and since $x \in \mathcal{A}^{*}$ we get that

$$
z k_{1} \in \mathcal{A}_{G^{+}}^{*} \text { or equivalently } z \in \mathcal{A}_{G^{+}}^{*}\left(K^{+}\right)^{-1} .
$$

We started with an arbitrary element $x \in \mathcal{A} \cap \mathcal{A}^{*}$, then we can conclude that

$$
\mathcal{A} \cap \mathcal{A}^{*} \subset L=K \operatorname{cl}\left(\mathcal{A}_{G^{+}}^{*}\right)\left(K^{+}\right)^{-1} .
$$

By hypothesis $\operatorname{cl}\left(\mathcal{A}_{G^{+}}^{*}\right)$ is compact and $\mathcal{A} \cap \mathcal{A}^{*}$ is dense in $\mathcal{C}$, thus $\mathcal{C}$ is contained in the compact set $L$, finishing the proof.

For simply connected nilpotent Lie groups we have the following: 
3.9 Corollary: Let $G$ be a nilpotent simply connected Lie group. Then $\mathcal{C}$ is bounded if, and only if, $\operatorname{cl}\left(\mathcal{A}_{G^{-}}\right)$and $\operatorname{cl}\left(\mathcal{A}_{G^{+}}^{*}\right)$ are compact subsets of $G$ and $\mathcal{D}$ is hyperbolic.

Proof: In fact, by Theorem $3.8 \mathcal{C}$ is bounded if and only if $G^{0}, \operatorname{cl}\left(\mathcal{A}_{G^{-}}\right)$and $\operatorname{cl}\left(\mathcal{A}_{G^{+}}^{*}\right)$ are compact subsets. Since $G$ is simply connected its exponential exp is a diffeomorphism which implies that $G^{0}$ is compact if and only if $\mathfrak{g}^{0}$ is compact if and only if $\mathfrak{g}^{0}=\{0\}$.

\subsection{Uniqueness}

In the classical Euclidean Abelian Linear Control Systems it is well known that under the Kalman rank condition there exists just one control set with non empty interior. We will show here that any solvable Lie group and any group $G$ such that $G^{0}$ is a compact subgroup have the same property, i. e., they have at most one control set. We start with,

3.10 Theorem: The set $\mathcal{C}$ is the only control set of the linear system (7) whose interior intersects $G^{+, 0} G^{-}$and $G^{-, 0} G^{+}$.

Proof: Let $\widetilde{\mathcal{C}}$ be a control set such that its interior intersects $G^{+, 0} G^{-}$and $G^{-, 0} G^{+}$. Since two control sets with nonempty intersection must coincide it is enough to show that $\mathcal{C} \cap \widetilde{\mathcal{C}} \neq \emptyset$. We will divide the proof in three steps:

Step 1: If int $\widetilde{\mathcal{C}} \cap G^{+, 0} G^{-} \neq \emptyset$ there are $\tau_{1}>0$ and $u_{1} \in \mathcal{U}$ such that

$$
a=\phi_{\tau_{1}, u_{1}} \in \operatorname{int} \widetilde{\mathcal{C}}
$$

Let $x \in \operatorname{int} \widetilde{\mathcal{C}} \cap G^{+, 0} G_{\widetilde{\mathcal{C}}}^{-}$and let $g \in G^{+, 0}$ and $h \in G^{-}$such that $x=g h$. Considering that in int $\widetilde{\mathcal{C}}$ controllability holds, there are $\tau>0$ and $u \in \mathcal{U}$ such that $\phi_{\tau, u}(x)=x$. Let $\varrho$ be a left invariant Riemannian metric on $G$. Since $\varphi_{t}(h) \rightarrow e$ as $t \rightarrow+\infty$ we have that

$$
\varrho\left(\phi_{t, u}(x), \phi_{t, u}(g)\right)=\varrho\left(\phi_{t, u}(g) \varphi_{t}(h), \phi_{t, u}(g)\right)=\varrho\left(\varphi_{t}(h), e\right) \stackrel{t \rightarrow+\infty}{\rightarrow} 0 .
$$

Since $\phi_{n \tau, u}(x)=x$, for any $n \in \mathbb{N}$, we obtain that $\phi_{t, u}(g) \in \operatorname{int} \widetilde{\mathcal{C}}$, for $t>0$ greater enough. Moreover, $g \in G^{+, 0} \subset \mathcal{A}$ which implies, by concatenation, that

$$
\phi_{\tau_{1}, u_{1}} \in \operatorname{int} \widetilde{\mathcal{C}} \text { for some } \tau_{1}>0, u_{1} \in \mathcal{U},
$$

as stated.

Step 2: If int $\widetilde{\mathcal{C}} \cap G^{-, 0} G^{+} \neq \emptyset$ there are $\tau_{2}>0$ and $u_{2} \in \mathcal{U}$ such that

$$
e \in \phi_{\tau_{2}, u_{2}}(\operatorname{int} \widetilde{\mathcal{C}})
$$


Let $x^{\prime} \in \operatorname{int} \widetilde{\mathcal{C}} \cap G^{-, 0} G^{+}$and $g^{\prime-, 0}, h^{\prime+}$ such that $x^{\prime}=g^{\prime} h^{\prime}$. Again by controllability in int $\widetilde{\mathcal{C}}$ there are $\tau^{\prime}>0, u^{\prime} \in \mathcal{U}$ such that $\phi_{-\tau^{\prime}, u^{\prime}}\left(x^{\prime}\right)=x^{\prime}$. Since $\varphi_{-t}\left(h^{\prime}\right) \rightarrow e$ as $t \rightarrow+\infty$ we have

$\varrho\left(\phi_{-t, u^{\prime}}\left(x^{\prime}\right), \phi_{-t, u^{\prime}}\left(g^{\prime}\right)\right)=\varrho\left(\phi_{-t, u^{\prime}}\left(g^{\prime}\right) \varphi_{t}\left(h^{\prime}\right), \phi_{-t, u}\left(g^{\prime}\right)\right)=\varrho\left(\varphi_{-t}\left(h^{\prime}\right), e\right) \stackrel{t \rightarrow+\infty}{\rightarrow} 0$.

Again, since $\varphi_{-n \tau^{\prime}, u^{\prime}}\left(x^{\prime}\right)=x^{\prime}$ for any $n \in \mathbb{N}$ we get that $\phi_{-t, u^{\prime}}\left(g^{\prime}\right) \in \operatorname{int} \widetilde{\mathcal{C}}$, for $t>0$ greater enough. Moreover, $g^{\prime-, 0} \subset \mathcal{A}^{*}$ which gives us by concatenation that for some $\tau_{2}>0, \Theta_{\tau_{2}} u_{2} \in \mathcal{U}$ we have

$$
\phi_{-\tau_{2}, \Theta_{\tau_{2}} u_{2}} \in \operatorname{int} \widetilde{\mathcal{C}} \Leftrightarrow e \in \phi_{\tau_{2}, u_{2}}(\operatorname{int} \widetilde{\mathcal{C}})
$$

Step 3: It holds that $\mathcal{C} \cap \widetilde{\mathcal{C}} \neq \emptyset$;

By (13) there is $b \in \operatorname{int} \widetilde{\mathcal{C}}$ such that $\phi_{\tau_{2}, u_{2}}(b)=e$. Let $a=\phi_{\tau_{1}, u_{1}} \in \operatorname{int} \widetilde{\mathcal{C}}$ given in (12). By controllability in int $\widetilde{\mathcal{C}}$ there are $\tau_{3}>0$ and $u_{3} \in \mathcal{U}$ such that $\phi_{\tau_{3}, u_{3}}(a)=b$. If we concatenate $u_{1}, u_{2}$ and $u_{3}$ and extend it periodically we obtain an admissible control function $u \in \mathcal{U}$ with period $T=\tau_{1}+\tau_{2}+\tau_{3}$. Moreover, $\phi_{t, u}(e)$ is a periodic solution of the linear system (17) passing by $a, b \in \operatorname{int} \widetilde{\mathcal{C}}$. Since periodic orbits cannot leave the interior of a control set we must have that it lies in $\mathcal{C} \cap \widetilde{\mathcal{C}}$ which concludes the proof.

As a direct corollary we have:

3.11 Corollary: If $G$ is a solvable Lie group with compact $G^{0}$-component, then $\mathcal{C}$ is the only control set.

Proof: If $G=G^{+, 0} G^{-}=G^{-, 0} G^{+}$it follows that any control set of (7) with nonempty interior is such that its interior intersects $G^{+, 0} G^{-}$and $G^{-, 0} G^{+}$. Consequently, by Proposition 3.1 item 5., if $G$ is a solvable Lie group or if $G^{0}$ is a compact subgroup, then $\mathcal{C}$ is the only control set of the linear system.

\section{References}

[1] V. Ayala And L.A.B. San Martin, Controllability properties of a class of control systems on Lie groups, Lecture Notes in Control and Information Sciences 258 (2001), pp. 83-92.

[2] Ayala, V. And J. Tirao, Linear control systems on Lie groups and Controllability, Eds. G. Ferreyra et al., Amer. Math. Soc., Providence, RI, 1999.

[3] Colonius, F. and C. Kliemann, The Dynamics of Control, Systems \& Control: Foundations \& Applications. Birkäuser Boston, Inc., Boston, MA, 2000.

[4] Da Silva, A. J., Controllability of linear systems on solvable Lie groups. To appear in SIAM, 2016. 
[5] Da Silva, A. J., Outer Invariance Entropy for Linear Systems on Lie Groups. To appear in SIAM (2014).

[6] Da Silva, A., Ayala, V., Controllability of linear systems on Lie groups with finite sensimple center. Submitted to the SIAM Journal of Control and Optimization, December 2015.

[7] Jounn, Ph., Controllability of Linear Systems on Lie group, Journal of Dynamics and Control Systems, 17 (2011) 591-616.

[8] Jounn, Ph. And Dath M., Controllability of Linear Systems on low dimensional Nilpotent and Solvable Lie groups, Journal of Dynamics and Control Systems (2014).

[9] Jounn, Ph., Equivalence of Control Systems with Linear Systems on Lie Groups and Homogeneous Spaces, ESAIM: Control Optimization and Calculus of Variations, 16 (2010) 956-973.

[10] Onishchik, A. L. And E. B. Vinberg, Lie Groups and Lie Algebras III - Structure of Lie Groups and Lie Algebras, Berlin: Springer (1990).

[11] San Martin, L. A. B., Algebras de Lie, Second Edition, Editora Unicamp, (2010). 